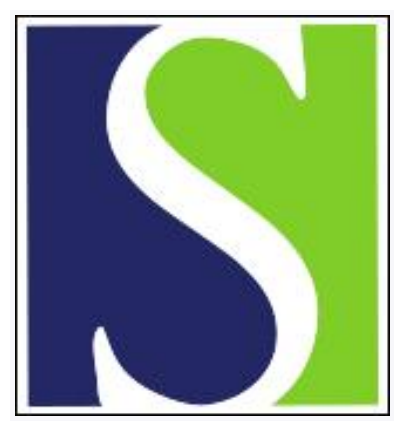

Scand J Work Environ Health 1990;16(1):55-58

https://doi.org/10.5271/sjweh.1815

Issue date: 1990

Psychophysical scaling with applications in physical work and the perception of exertion.

by Borg $G$

Affiliation: University of Stockholm, Sweden.

The following articles refer to this text: 2015;41(3):234-246;

2018;44(5):530-538; 2021;47(4):306-317

This article in PubMed: www.ncbi.nlm.nih.gov/pubmed/2345867

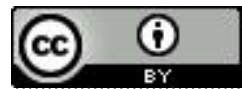




\title{
Psychophysical scaling with applications in physical work and the perception of exertion
}

\author{
by Gunnar Borg ${ }^{1}$
}

\begin{abstract}
BORG G. Psychophysical scaling with applications in physical work and the perception of exertion. Scand $J$ Work Environ Health 1990;16(suppl 1):55-8. In studies on work it is important to assess various subjective symptoms, complaints, and annoyances. To measure such symptoms, psychophysical ratio scales may be used, as along with simpler category rating scales. In this paper some of the basic concepts and methods of psychophysics have been described. In the field of heavy physical work and the perception of effort and exertion, one of the most popular methods is the rating of perceived exertion. This scale has been presented together with a new category ratio scale, commonly referred to as the CR-10 scale. Some situations in which it is important to obtain measurements of perceived exertion have also been described in the paper.
\end{abstract}

Key terms: physical effort, psychophysics, ratings of exertion, rating of perceived exertion, scales, subjective symptoms, work load.

To be in good health, happy, and satisfied is a very desirable goal. Nowadays people know that by improving their "life-style," they can improve their well being. They are not victims of destiny. Before their situation in work and leisure-time activities can be improved, it is of primary importance that they perceive the situation correctly. They must listen to the "signals of the body."

\section{What is psychophysics?}

The information obtained about the surrounding world and about bodily or environmental disturbances comes from one's sensory perception. A scientific subject that deals with these problems is psychophysics. It studies the detection and identification of objects and events, the discrimination of stimuli, and scaling, ie, studies of the relations between perceptual and physical intensities.

\section{Why scaling?}

There are several reasons why it is important to scale subjective aspects of such physical stress as the perception of exertion and discomfort. One reason is general scientific interest. This interest refers to human curiosity and the need to better understand the surrounding world. Another reason is that sensory organs and conscious perceptions reveal important disturbances in the environment. In this way a person may get important information about disturbances that are not so easy to measure physically or by physiological

\footnotetext{
I University of Stockholm, Stockholm, Sweden.
}

Reprint requests to: Professor G Borg, University of Stockholm, S-106 91 Stockholm, Sweden. reactions. Still another fundamental reason is that perceptions reveal disturbances within a person by subjective symptoms that refer to early signs of diseases.

\section{Units in measurements}

The main problem in psychophysics is the lack of fundamental units for measuring perceptual intensities. "Objective" physical scales have units that are well defined, constant, and public. When the term "subjective" is used, it often means that there is both uncertainty and privateness. One challenge in psychophysical scaling is then to improve the methods so that they come closer to the requirements of a good scale, ie, so that the uncertainty and the variability of the unit is diminished and so that it becomes "semipublic" to permit interindividual comparisons.

Another requirement of the unit should be added, namely, it should be "meaningful." Before modern standardizations, units were chosen from everyday life, eg, "foot" and "horsepower." They were lacking in precision and constancy, but they had a "surplus meaning" to people. (It is an irony of fate that Watt himself proposed horsepower as the unit for power.) The units of psychophysical ratio scales also lack meaning for direct "level" estimates. This is a significant drawback that has not been recognized as it should.

\section{Psychophysical ratio scaling methods}

In psychophysical ratio scaling the scientist's goal is to use methods that have the same metric qualities as methods used in physics and physiology, ie, methods with an absolute zero and with the same distances between the scale values. Some of these techniques are estimation methods according to which the subjects are asked to estimate given stimuli and assign them 
numbers. Others are adjustment methods according to which the subjects have to set a certain stimulus intensity so it is perceived to be, eg, half or twice as intense as a certain standard stimulus. One of the most popular ratio scaling methods is "magnitude estimation" (1). The subjects are asked to assign numbers to stimuli of different intensities in such a way that the numbers given match the perceived intensities and vary directly in proportion to them.

To demonstrate how a ratio scaling method may function, consider ratio production and the perception of speed while driving a car. The subject drives 100 $\mathrm{km} / \mathrm{h}$ and is instructed to decrease the speed until it is perceived to be half as fast. The actual speed is really about $70 \mathrm{~km} / \mathrm{h}$, which is very much above the physical half. Several different intensity levels must be used in the experiment, and the results must show the same relation between intensities, ie, $50 \mathrm{~km} / \mathrm{h}$ should be decreased to 35 to be subjectively half.

The perception of effort and exertion follows the same positively accelerating function as the perception of speed. When the physical work load is decreased until it is perceived to be half as heavy "subjectively," it is really still more than half as intense "objectively." The opposite is the case with the perception of loudness and noise. See figure 1.

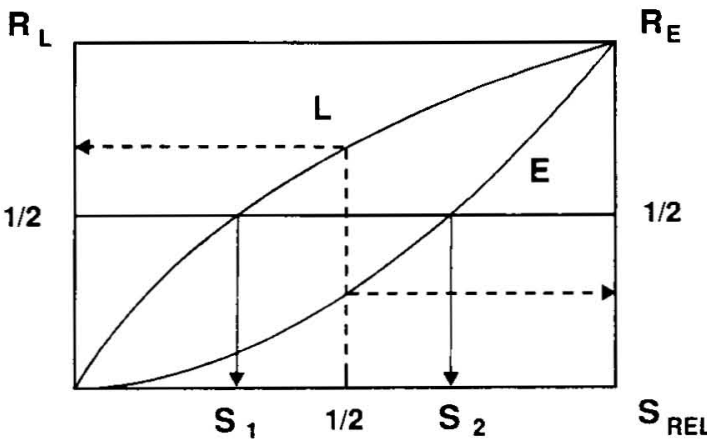

Figure 1. Manner in which the perception (R) of loudness (L) and exertion (E) grows with the physical intensity (S) [S in a relative (REL) scale] and what happens when the intensity is halved. See the text.

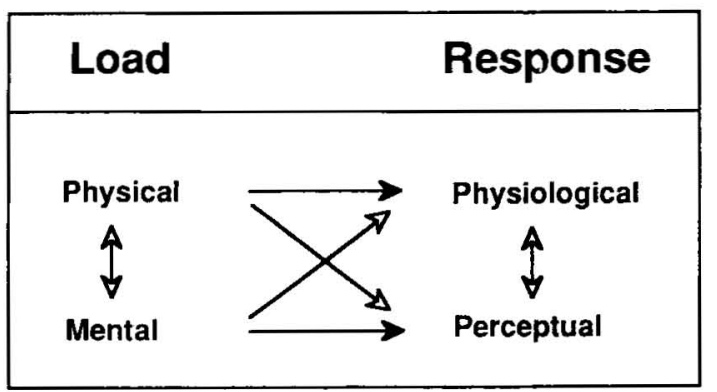

Figure 2. Illustration of the fact that physiological and perceptual responses should be used as indicators of both physical and mental work load.
- Most psychophysical relations may be mathematically described by power functions. In the preceding example, a power function with an exponent of about two describes how the subjective speed grows to the approximate square of the physical speed (2). In the scaling of visual brightness an exponent of 0.3 has been found, and for loudness the exponent is about 0.6 .

\section{Perception of exertion in physical work}

Ratings of perceived exertion (or "effort and exertion') are important complements to physiological measurements. Of all single indicators of the degree of physical strain, perceived exertion is one of the most informative. It integrates a great amount of information, with cues from the peripheral muscles and joints, cardiovascular and respiratory functions, and the central nervous system.

In many studies on work, interest has been focused too much on mere performance. However, "the costs" behind the performances in the form of physiological and perceptual measurements must, of course, also be taken into account before men and women can be better understood at work. Thus it is necessary to integrate the information obtained from the three different kinds of "effort variables," ie, performances, physiological measurements, and perceptual responses.

Figure 2 shows that perceptual estimations of physical and mental load complement the physiological measurements. Physical load is often studied with physiological responses and mental load with perceptual ones. In many studies of mentally stressing situations it is common to use physiological indicators of the degree of strain. It is, however, equally important to use perceptual indicators of physical strain.

\section{Ratio scaling of perceived exertion}

The first psychophysical studies on perceived exertion in heavy physical work were done by Dahlström and myself. [See reference 2.] Both short-term work (less than 1-min) and work of longer duration (several minutes) were studied for exercise on the bicycle ergometer. Ratio scaling methods were applied and showed that the perception of exertion grows according to positively accelerating functions with the physical work load (power). Mathematically these functions could be described by power functions with an exponent of about 1.6. In one of the most recent studies (3), positively accelerating functions of about the same appearance were found for running and biking (both leg work and arm cranking), while walking showed a stronger positively accelerating function. The relations between estimations of perceived exertion according to two different methods and heart rates and blood lactate concentrations were the same, however, independent of the kind of work performed. The increase in perceived exertion could very well be predicted from 
a combination of the increases in heart rate and blood lactate concentrations.

A very useful mathematical expression that makes it possible to describe both psychophysical and physiological intensity variations in the same way is the following general power function:

$$
R=a+c(S-b)^{n}
$$

where $\mathrm{R}$ is the intensity of the response, $\mathrm{S}$ is the stimulus intensity, $\mathrm{a}$ and $\mathrm{b}$ are constants showing the starting point of the function (or the absolute threshold), $\mathrm{c}$ is the measure constant, and $\mathrm{n}$ is the exponent (2).

\section{The RPE scale}

The aforementioned ratio scaling methods are very good methods when one wants to describe how the subjective intensity varies with the physical intensity. They do not, however, give any valid intensity levels for differential use. To say that one weight is twice as heavy as another does not say if it is heavy or not. For practical purposes a simple category rating scale (the RPE scale, ie, the scale for rating of perceived exertion, see figure 3 ) was therefore constructed $(4,5)$. The scale was designed to grow linearly with exercise intensity and heart rate for work on the bicycle ergometer. If one does not know exactly how to construct a rating scale, this is a meaningful way to do so, even if it violates the true growth of perceived exertion. The scale has been translated into many different languages and has become internationally popular for the evaluation and monitoring of exercise intensities.

\section{The category ratio scale}

In recent years a new category ratio (CR) scale has been developed to meet the twofold demands of ratio scaling and level estimations. In the CR scale, the verbal expressions are anchored to the correct positions on a ratio scale according to their quantitative meaning (6). In the simplest version of this scale, numbers from 0 to 10 are used (figure 4 ). The number 10 implies an extremely strong perceptual intensity, as in the perceived intensity in extremely heavy physical exercise like running for some minutes as fast as possible or lifting and carrying extremely heavy weights. Ten is defined as the strongest effort and exertion a person has ever experienced. Since he or she, however, may imagine an intensity that is even stronger, the "absolute" maximum is placed somewhat higher. The subject may thus use 12,13 or an even higher number. This is specially true for the perception of aches and pain, where "maximal" normally lies above the strongest perception of exertion.

One very special and important property of the CR scale is that, by anchoring the highest number in a very well-defined perceived effort and exertion with a de-

$\begin{aligned} & \text { Borg's RPE scale } \\ 6 & \text { No exertion at all } \\ 7 & \text { Extremely light } \\ 8 & \text { Very light } \\ 9 & \\ 10 & \\ 11 & \text { Light } \\ 12 & \\ 13 & \text { Somewhat hard } \\ 14 & \\ 15 & \text { Hard } \\ 16 & \\ 17 & \text { Very hard } \\ 18 & \\ 19 & \text { Extremely hard } \\ 20 & \text { Maximal exertion }\end{aligned}$

Figure 3. The RPE scale, ie, the 15-grade scale for ratings $(R)$ of perceived $(P)$ exertion $(E)(4,5)$.

Borg's CR-10 scale

$0 \quad$ Nothing at all

0.5 Extremely weak (just noticeable)

1 Very weak

2 Weak

(light)

3 Moderate

4

$5 \quad$ Strong

(heavy)

6

$7 \quad$ Very strong

8

9

10 Extremely strong (almost max)

\section{Maximal}

Figure 4. The CR-10 scale, ie, the category (C) scale with ratio (R) properties (6).

gree of "sameness" for different individuals, a good point of reference is obtained. One can then use this value or part of it as a "semi-public" unit for many different kinds of interindividual and intermodal comparisons, such as between noise, vibration, pain, taste, and exertion for different groups of people. 


\section{Applied studies}

That it is important in many work situations to obtain estimations of the degree of physical strain and discomfort and complaints is fairly self-evident. In a recent document of the International Organization for Standardization, it is also stated that: "Account shall be taken of objectively measurable phenomena and of subjective assessments [See paragraph 4.2]" (7). This statement shows how important it is to use "standardized" methods for evaluating subjective symptoms.

Many studies have been performed with the use of the RPE scale. Other methods for studying the perception of physical strain and fatigue have also been used. Among these are various rating methods and methods of adjustment to obtain "acceptable work loads" in manual materials handling. These studies have, however, been treated in another paper (8).

In differential studies of perceived exertion high correlations have been found not only with heart rates and blood lactate concentrations but also with many other physiological variables both in reference to peripheral and central cues. One of the most common uses of the RPE scale is the clinical diagnosis of heart patients and patients with respiratory disturbances. The normal growth pattern and the level of exertion changes dramatically in different clinical populations. The RPE scale is also used in rehabilitation and for the prescription and regulation of exercise intensities or as a means to evaluate a certain training situation. A similar use is also found in epidemiologic studies for intensity evaluations of different work tasks and leisure-time activities, as a complement to the intensity evaluations that can be performed from kind of work tasks and from duration and frequency measurements.

In ergonomic investigations of work tasks perceived exertion has been used in studies of heavy aerobic work. The value of subjective estimations is also evident in job situations where the work tasks consist of short-term static work for which valid physiological measurements are difficult to obtain.

One of the first investigations in which different kinds of work operations were studied was performed by Gamberale (9). The investigation showed that, for work on a bicycle ergometer, a weight-lifting task, and a task consisting of pushing a wheelbarrow, the subjects could very well discriminate between the overall perceived exertion, exertion in the legs, and exertion in the arms. The study also showed that these perceptual differences could be partially "explained" by the differences in oxygen consumption, heart rate, and blood lactate concentrations. Work tasks in many different kinds of work have been studied, eg, in operations performed by people in various industrial occupations, by cleaners, truck drivers, etc.

For further studies the reader is referred to the proceedings from a symposium on the perception of exertion in physical work (10).

\section{Concluding remarks}

The interest in attending to and interpreting subjective symptoms for different people and different work tasks has increased during recent years. Psychophysical methods developed to measure sensory perceptions can be used to determine most kinds of subjective responses referring to disturbances in the environment or in the body itself. One important class of symptoms is the somatosensory perceptions of effort and exertion in heavy physical work. When ratio scaling methods are used to measure perceived exertion, functions for various work loads are obtained which can be compared with physiological responses, such as heart rate and blood lactate concentration. However, when direct intensity levels of the degree of perceived exertion is wanted, simple category rating methods are more informative than the ratio scaling methods. Of special interest is the new category ratio scale that permits determinations of psychophysical growth functions and allows direct level estimations to be obtained for the evaluation of ergonomic work tasks.

\section{Acknowledgments}

The preparation of this paper was made possible thanks to a research grant from the Swedish Council for Research in the Humanities and Social Sciences (F561/88).

\section{References}

1. Stevens SS. Matching functions between loudness and ten other continua. Percept Psychophys 1966;1:5-8.

2. Borg G. Physical performance and perceived exertion. Lund (Sweden): Gleerup, 1962. (Studia psychologica et pædagogica, series altera, investigations XI.)

3. Borg G, van den Burg M, Hassmén P, Kaijser L, Tanaka S. Relationships between perceived exertion, HR and HLa in cycling, running and walking. Scand J Sports Sci $1987 ; 9: 69-77$

4. Borg G. Perceived exertion as an indicator of somatic stress. Scand J Rehabil Med 1970;2:92-8

5. Borg G. An introduction to Borg's RPE-scale. Ithaka, NY: Mouvement Publications, 1985.

6. Borg G. A category scale with ratio properties for intermodal and interindividual comparisons. In: Geissler HG, Petzold P, ed. Psychophysical judgment and the process of perception. Berlin: VEB Deutscher Verlag der Wissenschaften, 1982:25-34.

7. International Organization for Standardization. Ergonomic principles in the design of work systems. Geneva: International Organization for Standardization, 1981. (ISO 6385.)

8. Gamberale F. Perception of effort in manual materials handling. Scand J Work Environ Health 1990;16(suppl 1):59-66.

9. Gamberale F. Perceived exertion, heart rate, oxygen uptake and blood lactate in different work operations. Ergonomics 1972;15:545-54.

10. Borg G, Ottoson D, ed. The perception of exertion in physical work. London: McMillan Press, 1986. (WennerGren Center international symposium series; vol 46.) 